Results A 23 year Caucasian lady was referred to lymphoma clinic by her GP with a three month history of lymphadenopathy and fatigue. She was diagnosed with a primary CMV infection and lost to follow up after her symptoms resolved. One year later she attempted to donate blood. Positive syphilis serology with a low RPR was detected on routine blood screening by the transfusion service. The patient was referred to Bristol Sexual Health Centre where sexual history taking revealed she had a bi sexual partner at the time of her illness who also tested positive for syphilis. An archived blood sample from the time of her illness revealed active syphilis infection with a high RPR.

Discussion/conclusion Secondary syphilis can mimic numerous illnesses. However syphilis testing remains uncommon outside of sexual health clinics for a variety of reasons. A change of culture is required to ensure medical professionals are prepared for routine sexual enquiry and consider syphilis as a potential cause for lymphadenopathy.

\section{P234 EXPLORING SERVICE USER NEEDS AND STREAMLINING DELIVERY THROUGH TRIAGE BY SENIOR CLINICIANS IN AN INTEGRATED SEXUAL HEALTH WALK-IN}

Sophia Davies, Rachel Amherst*, Jane Bush. Northern Devon Healthcare Trust, Exeter, UK

\subsection{6/sextrans-2016-052718.282}

Background/introduction On-going procurement exercises in Sexual Health inevitably require that efficiencies are sought whilst maintaining high quality and equity of access.

Aim(s)/objectives This test of change aimed to inform a streamlining of a city centre walk-in service and to test the feasibility of delivering an integrated service using staff with mixed skill sets. Means were sought to reduce re-attendances to the colocated Contraception and GU Medicine services.

Methods For two weeks in January 2016 patients attending the walk-in service were triaged by a senior dual trained clinician. Triage notes were made and patients were subsequently seen by another clinician who could meet all of their needs. Data was collected regarding staff skillsets, waiting times, patient-perceived versus clinician-assessed needs as well as services actually delivered and staff feedback.

Results 324 patients were seen (236 females, 88 male). Approximately $40 \%$ of women had mixed (GU and contraception) needs, whilst of those believing themselves to have a solely contraception need, half were identified as also having a GU need. A third of patients were symptomatic Two thirds had a sexual health screen. Nursing staff saw $66 \%$ of patients. $80 \%$ of staff reported similar or increased job satisfaction (qualitative data are available).

Discussion/conclusion Service users perceived needs are often less than those assessed by experienced clinicians. Delivering an integrated walk-in service whilst maintaining specialist skills is feasible if triage is effective; missed opportunities are minimised. Work to assess the utility and cost-effectiveness of different types of triage is required

\section{P236 A REVIEW OF A YEAR OF NEW HIV DIAGNOSES AT A SINGLE CENTRE - WHAT CAN WE LEARN? HOW PEOPLE TEST, PRIMARY INFECTION AND HOSPITALISATION}

${ }^{1}$ Sarah Cavilla* ${ }^{1,2}$ Daniel Richardson. 'Brighton \& Sussex University Hospitals NHS Trust, UK; ${ }^{2}$ Brighton \& Sussex Medical School, Brighton, UK

10.1136/sextrans-2016-052718.283
Introduction A large proportion of new HIV diagnoses are incident and hospitalised. HIV testing can be accessed by various means. HIV incidence in UK men who have sex with men (MSM) continues to increase. HIV testing is an important public health intervention to reduce incidence. Locally we have a high prevalence $(8: 1000)$ and an HIV cohort of 2300 mainly MSM.

Methods New diagnoses from January- December 2015 were identified from our local database, clinical records were examined. Data was collected on patient characteristics, medical history, results, the method and location of testing.

Results There were 57 new diagnoses; 50 (88\%) MSM, 4 (7\%) heterosexual females and $3(5 \%)$ heterosexual males. $24(42 \%)$ had tested negative in the previous year, $24(42 \%)$ more than 1 year ago and $9(16 \%)$ had never tested. Testing locations included: 27 (47\%) GUM clinic, 14 (25\%) GP, 5 (9\%) home test kits, 4 (7\%) THT, 3 (5\%) inpatient, 2 (3.5\%) hospital outpatients, $1(1.75 \%)$ sauna and $1(1.75 \%)$ private clinic. Reasons for testing encompassed: 17 (30\%) GUM screening, 10 (18\%) HIV symptoms, 2 (4\%) post exposure prophylaxis, 2 (4\%) partner notification, 2 (4\%) malignancy, 1 (2\%) assault, 1 (2\%) condom break, $1(2 \%)$ intravenous drug use and $1(2 \%)$ insurance. 12/49 (24\%) avidity results were incident. 10/57 (18\%) were hospitalised within 3 months of diagnosis; 3 malignancies, 2 opportunistic infections (Pneumocystis, Mycobacterium avium complex), 1 STI (Shigella), 1 abscess, 1 seroconversion, 1 treatment toxicity and 1 for investigations.

Discussion/conclusion Locally a large proportion of new HIV diagnoses are incident, MSM and hospitalised. Continued innovation is vital in community and hospital admission testing to reduce undiagnosed HIV and incidence.

\section{P237 ASSESSING THE IMPACT OF A HIV TESTING POLICY}

${ }^{1}$ Sarah Allstaff*, ${ }^{2}$ Ewan Barrack, ${ }^{1}$ Ciara Cunningham. ${ }^{1}$ NHS Tayside, Dundee, UK; ${ }^{2}$ University of Dundee, Dundee, UK

\subsection{6/sextrans-2016-052718.284}

Background/introduction Mirroring national data, HIV testing in our local board is failing to reduce the high proportion of late diagnoses. Healthcare Improvement Scotland (HIS) HIV standards 2011 recommended the development and promotion of a written HIV testing policy. A local HIV testing policy was introduced in 2013 based on the UK National testing guideline (BHIVA), recommending routine testing in certain clinical areas, in high risk groups and all individuals with "clinical indicator conditions" regardless of perceived risk. The introduction of the policy was supported by staff training.

Aim(s)/objectives Review the impact of a HIV testing policy on staff knowledge and levels of HIV testing.

Methods In November 2015 a survey was undertaken to question staff awareness of the policy, understanding of HIV testing and levels of HIV testing. The survey was advertised on local websites to healthcare, social work, third sector and substance misuse staff.

Results The survey had 120 respondents, with the largest proportion from Consultant and GP staff. Over $70 \%$ of respondents were aware of the policy. $25 \%$ reported that the policy had changed their clinical practice by increasing their confidence in testing. There has been a corresponding increase in HIV testing figures. Interestingly over $70 \%$ responded that a detailed HIV risk assessment was required even in the presence of a clinical indicator condition. 
Discussion/conclusion The survey has demonstrated that the HIV testing policy has increased staff awareness and confidence in HIV testing. Late diagnosis rates continue to be monitored and misconceptions regarding HIV testing challenged.

\section{P239 DON'T ASK, DON'T TELL: INTRODUCTION OF ROUTINE DOMESTIC ABUSE PROMPT IN GENITOURINARY WALK- IN CLINIC}

Marie Williamson*, Frances Beanland, Andrea Mauger, Rachel Sacks, Alison Mears. Imperial College Healthcare NHS Trust, London, UK

\subsection{6/sextrans-2016-052718.285}

Background/introduction Domestic abuse (DA) is common and spontaneous disclosure rare. In July 2015, routine DA enquiry was introduced in a busy, inner London, walk-in genitourinary medicine clinic. All DA disclosures prompted completion of a proforma identifying ongoing risk/ $<3 / 12$ (=current DA) with clear management pathway. Patients disclosing current DA were seen by Sexual Health Information Protection (SHIP) team for detailed risk assessment and referrals, as needed. Those with previous DA were offered information/referrals if indicated.

Aim(s)/objectives To review DA disclosure management following DA routine prompt introduction.

Methods A retrospective case-notes review of patients' notes (coded DA) for 3 months from 14/07/2015.

Results 137 patients (111 women,26 men): age-range 17-75 years old(mean 33), 7\% (10/137) commercial sex workers, $11 \%$ (15/137) MSM/bisexual. $72 \%$ of patients had never disclosed to professionals previously. Ex-regular and current partners most common perpetrators (62\%, 15\% respectively). 25\% (34/137) disclosed current DA. Of these, 94\% (32/34) were offered SHIP referral, 88\% (28/32) accepted, 75\% (21/28) were seen. Referrals were made to DA services $48 \%(10 / 21)$, refuges $10 \%(2 / 21)$, MARAC 19\% (4/21). 52\% (11/21) had a standardised risk assessment; those who did not, 60\% (6/10) were already engaged in services, 20\% (2/10) didn't attend follow-up and $20 \%(2 / 10)$ referred to psychology. 44\% (14/34) patients were discussed at Vulnerable Adults MDT. No escalation of violence was recorded.

Discussion/conclusion Patients with significant risk from DA were identified who may not have disclosed without routine prompt. This is suggested as the majority were first disclosures. Interventions to reduce the negative consequences of DA for these patients have been performed.

\section{P240 WHAT IS THE HEPATITIS B VACCINATION COVERAGE IN MSM IN SOUTH WEST LONDON? AN AUDIT OF HEPATITIS B VACCINATION COVERAGE IN 'FIRST ATTENDEE' MSM IN A BUSY TEACHING HOSPITAL GUM CLINIC}

\footnotetext{
${ }^{1,2}$ Rachel Hill-Tout*, ${ }^{2}$ Holly Mitchell, ${ }^{2}$ Gwenda Hughes. ${ }^{1}$ St George's NHS Foundation Trust, London, UK; ${ }^{2}$ Public Health England, London, UK
}

\subsection{6/sextrans-2016-052718.286}

Background The 2001 National Strategy for Sexual Health and HIV recommended 90\% uptake of Hepatitis B (HBV) vaccine in non-immune MSM at first GUM clinic attendance. The HepB3 Survey reported 95\% uptake in 2008 but recent surveillance using GUM Clinic Activity Dataset-v2 (GUMCADv2) coding shows $<20 \%$ uptake. A detailed regional audit was designed to investigate this apparent drop in coverage.

Aim To determine HBV vaccination coverage in 'first-attendee' MSMs.

Methods All MSM 'first-attendees' at our service between January-March 2014 were identified. Patient records were reviewed for HBV screening, vaccine-offer, vaccine-uptake, HIV testing and coding accuracy up to 18 months from first-attendance. MSM were deemed 'immune' if surface antibody (sAb) $>10$ $\mathrm{mIU} / \mathrm{ml}$, core antibody positive, or self-reported vaccinationstatus was 'Fully-vaccinated' and no serology was done; and eligible for vaccination if $\mathrm{sAb} \leq 10 \mathrm{mIU} / \mathrm{ml}$, or if they reported 'Partially-vaccinated', 'Never-vaccinated', or 'Don't know' and no serology was done.

Results We identified 115 MSM 'first-attendees' (13 HIV+). 41\% only attended once. Regarding vaccination-status: 41/95 (43\%) reported 'Fully-vaccinated', $29 / 95$ (30\%) 'Partially-vaccinated', 12/95 (13\%)'Never-vaccinated', 11/95 (12\%) 'Don'tknow', 1/95 (1\%) 'Chronic-HBV' and 1/95 (1\%) 'Cleared-HBV'. 48/103 (47\%) were deemed immune and 46/103 (45\%) eligible. $36 / 46(76 \%)$ of eligibles were offered vaccination; 2/36 (6\%) declined, reporting 'not at risk'. $3 / 32$ (9\%) who accepted vaccination pending sAb levels did not return for it. 31/46 (67\%) of eligibles received $\geq 1$ dose of vaccine, 28/46 (61\%) within 42 days of first-attendance. Reasons for non-offer were not recorded. $75 \%$ of first-doses were coded. Only 15\% of 'immune' patients were coded as such (P2I). HIV-test uptake was $99 \%$ and coding accuracy was $97 \%$.

Discussion We found below-target levels of HBV vaccinationcoverage and incomplete coding of immunity/vaccination. Failure to code P2I for 'immunes' will increase the apparent 'eligibles' denominator in GUMCADv2 algorithms, generating incorrectly low vaccination-coverage figures. Reduced offer-rate may contribute to low vaccination-coverage and should be reviewed locally. Further regional audits may be required. Significant improvements in coding are essential for accurate surveillance of HBV vaccination-coverage using GUMCADv2.

\section{P241 DO MEN ATTENDING A GENITOURINARY MEDICINE SERVICE KNOW IF THEIR MOST RECENT SEXUAL PARTNER WAS USING CONTRACEPTION?}

William Gibson*, Amy Pearce, Frances Keane. Royal Cornwall Hospital, Truro, UK

\subsection{6/sextrans-2016-052718.287}

Background/introduction Unintended pregnancy is a significant problem. In 2014 in England and Wales 184,571 abortions were performed. Contraceptive methods generally focus on females. Males have the potential to contribute significantly to the contraceptive decisions in their relationships.

Aim(s)/objectives To demonstrate what knowledge male patients have of their partner's contraception status.

Methods Electronic proformas for male GUM patients in the region studied were amended to include a question that assessed whether or not the attending patient was aware if their most recent sexual partner (MRSP) was using contraception. After this was in place, notes of all male patients classified "new"/"rebook" who attended in January 2016 were studied. Those coded MSM (men who have sex with men) were excluded. An Excel workbook was created from the data of the 396 patients. Patients assessed on alternative proformas were excluded, as were those 